\title{
COMPOSIÇÃO FLORÍSTICA DE UM FRAGMENTO DE MATA CILIAR NA BACIA HIDROGRÁFICA DO RIO CABELO, JOÃO PESSOA, PARAíBA, BRASIL
}

\author{
FLORISTIC COMPOSITION OF A FRAGMENT OF RIPARIAN VEGETATION IN \\ THE RIO CABELO RIVER BASIN, JOÃO PESSOA, PARAÍBA, BRAZIL
}

Luiz de Aquino Pereira

Earl Celestino de Oliveira Chagas ${ }^{2}$

Maria Regina de V. Barbosa ${ }^{3}$

\section{Resumo}

Este estudo teve como objetivo conhecer a composição florística de um remanescente de mata ciliar ao longo do Rio Cabelo, e seu grau de similaridade com outros remanescentes florestais da região metropolitana de João Pessoa. Foram identificadas 151 espécies, distribuídas em 123 gêneros, pertencentes a 63 famílias. Do total de espécies amostradas, 54 (36\%) são árvores, 27 (17\%) arbustos, 39 (26\%) ervas, 26 (18\%) trepadeiras e $5(3 \%)$ parasitas. A comparação florística evidenciou acentuada heterogeneidade e baixos índices de similaridade entre os fragmentos comparados.

Palavras-chave: Mata ciliar, Flora, Nordeste do Brasil

\begin{abstract}
This study aimed to know the floristic composition of a remnant riparian forest along Rio Cabelo and the degree of similarity between that and other forest remnants in the metropolitan area of João Pessoa. We identified 151 species in 123 genera, belonging to 63 families. Of all species, 54 (36\%) are trees, $27(17 \%)$ shrubs, $39(26 \%)$ herbs, $26(18 \%)$ vines and $5(3 \%)$ parasites. The floristic comparison showed marked heterogeneity and low levels of similarity between fragments compared.
\end{abstract}

Key words: Riparian forest, Flora, Northeastern Brazil

\footnotetext{
1 Programa de Pós-Graduação em Biologia Vegetal, Universidade Federal de Pernambuco, Recife-PE. E-mail: luizdeaquinopereira@gmail.com

2 Programa de Pós-Graduação em Biologia Vegetal, Universidade Federal de Pernambuco, Recife-PE. E-mail: earlchagas@gmail.com

3 Departamento de Sistemática e Ecologia, Universidade Federal da Paraíba, João Pessoa-PB. E-mail: mregina@dse.ufpb.br
} 


\section{INTRODUÇÃO}

As matas ciliares são formações vegetacionais que ocorrem associadas às margens de diversos corpos d'água. Estas desempenham importantes funções ecológicas, como a estabilização das encostas dos rios, disponibilização de alimento e micro-hábitat para espécies aquáticas, manutenção da qualidade da água, e estabilização térmica da rede hidrográfica (HINKEL, 2003). Além disso, facilitam o deslocamento da fauna associada já que se constituem como um eficiente corredor ecológico (LIMA e ZAKIA, 200o).

Mesmo sendo áreas legalmente protegidas, essas matas continuam sendo intensamente devastadas em várias partes do Brasil, principalmente pelo avanço da exploração agropecuária e o estabelecimento de centros urbanos ao longo de sua área de ocorrência. Contudo, essas formações ciliares são caracterizadas por baixos valores de similaridade florística e uma grande diversidade de espécies (RODR IGUES e NAVE, 2000).

O remanescente de mata ciliar ao longo do Rio Cabelo foi escolhido para o desenvolvimento deste trabalho por ser uma das áreas prioritárias para a conservação e recuperação no município de João Pessoa. Essas áreas foram propostas no Plano Municipal de Conservação e Recuperação da Mata Atlântica, desenvolvido pela Prefeitura Municipal em parceria com a Fundação SOS Mata Atlântica em 2012 (SEMAM, 2012).

Dessa forma este trabalho objetiva subsidiar as ações preservacionistas previstas no plano, contribuindo para ampliar o conhecimento acerca da composição florística da área e avaliar sua similaridade com outros remanescentes da região metropolitana de João Pessoa.

\section{MATERIAL E MÉTODOS}

O trabalho foi realizado em um fragmento de mata ciliar localizado na bacia hidrográfica do Rio Cabelo (figura 1), litoral sul da cidade de João Pessoa, entre as coordenadas $7^{\circ} \mathrm{O} 8^{\prime} 53^{\prime \prime}$ e $7^{\circ} 11^{\prime} \mathrm{O} 2^{\prime \prime}$ de latitude sul e $34^{\circ} 47^{\prime} 26^{\prime \prime}$ e $34^{\circ} 50^{\prime} 33^{\prime \prime}$ de longitude oeste. A área apresenta altitude média de 31,15m (LEITE, 2005), latossolos (SASSI et al. 1997), clima tropical quente-úmido com temperaturas médias em torno de $25^{\circ} \mathrm{C}$ e precipitação de $1800 \mathrm{~mm}$ anuais (OLIVEIRA, 2001). Desde a década de 1980 a área vem sendo submetida a um intenso processo de urbanização e retirada exploratória de areia para construção civil, que tem gerado grandes supressões da vegetação ciliar. 


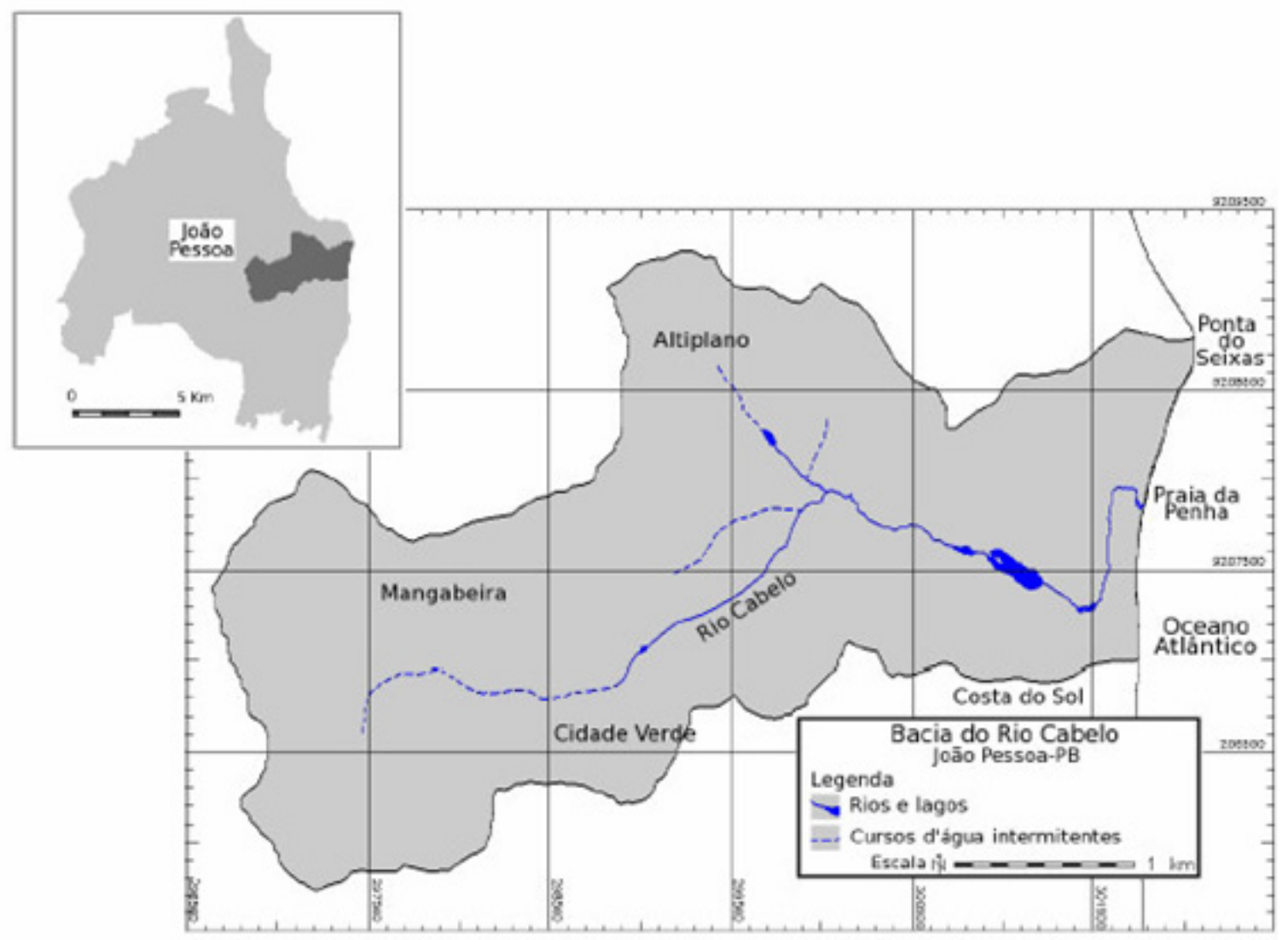

Figura 1. Localização da bacia hidrográfica do Rio Cabelo no município de João Pessoa/PB. Fonte: Farias et al., 2008

Foram realizadas seis expedições de campo entre os meses de outubro de 2010 a abril de 2011. Os espécimes foram coletados através de caminhadas aleatórias ao longo da vegetação que margeia o Rio Cabelo. A área considerada como mata ciliar foi estipulada de acordo com os dispostos na Lei no 4.777/65, que determina a área ciliar de acordo com a largura do corpo d'água, de forma que para o Rio Cabelo esta área corresponde a $30 \mathrm{~m}$ a partir da margem do rio, já que sua calha não ultrapassa os 10 m (LINDNER, 2003). Todo material foi processado no Laboratório de Taxonomia de Angiospermas (TAXON), no Departamento de Sistemática e Ecologia da Universidade Federal da Paraíba, conforme técnicas usuais de herborização (MORI et al. 1989).

O material coletado foi identificado com base em literatura especializada e através de comparações com materiais de herbário previamente identificados por especialistas. Todas as exsicatas foram montadas e incorporadas à coleção do Herbário Professor Lauro Pires Xavier (JPB). A grafia dos nomes das espécies e a abreviação dos nomes dos autores foram baseadas na Lista de Espécies da Flora do Brasil (FORZZA et al. 2013). Para a elaboração da lista florísticas foi adotada a proposta do APG III (2009).

Na comparação florística foi calculado o IS - Índice de similaridade de Jaccard (MUELLER-DOMBOIS e ELLEMBERG, 1974) entre a flora deste levantamento e a de outras quatro áreas da região metropolitana de João Pessoa. Os critérios utilizados 
para a escolha das áreas de mata ciliar (AMAZONAS, 2011; FREITAS, 2011) foram baseados na existência de um levantamento florístico pré-existente para a área e a matriz vegetacional (Floresta Estacional Semidecidual) em que estão inseridas. No caso das áreas não ciliares (BARBOSA, 2008; BARBOSA et al. 2009) além dos pré-requisitos anteriores sua escolha foi motivada a fim de verificar se a composição florística das matas ciliares da região metropolitana de João Pessoa possui um conjunto de espécies características destas formações. O IS Jé expresso pela fórmula:

$\mathrm{IS}_{\mathrm{J}}=[\mathrm{c} /(\mathrm{A}+\mathrm{B}-\mathrm{c})] \times 100$

Onde: IS $_{J}$ - Índice de Similaridade de Jaccard;

c - Número de espécies comuns entre as duas áreas;

A - Total de espécies presentes na área A;

B - Total de espécies presentes na área B;

\section{RESULTADOS E DISCUSSÃO}

Foram registradas 151 espécies distribuídas em 123 gêneros e 63 famílias botânicas, apresentadas na tabela 1 . As famílias com maior riqueza de espécies foram Fabaceae (14), Rubiaceae (12), Euphorbiaceae, Myrtaceae e Poaceae (8), que juntas abrigam $33 \%$ do total de espécies. O gênero mais diverso foi Eugenia com quatro espécies, seguido de Myrcia, Inga, Solanum, Polygala, Scleria e Panicum, todos com três espécies.

Tabela 1. Lista das espécies da mata ciliar da bacia hidrográfica do rio Cabelo, João Pessoa-PB. Arv - árvore; Arb - arbusto; Erv - erva; Tre - trepadeira e Para - parasitas.

FAMÍLIA/ESPÉCIE

Acanthaceae

Aphelandra sp.

Ruellia paniculata L.

Anacardiaceae

Anacardium occidentale L.

Tapirira guianensis Aubl.

Thyrsodium spruceanum Benth.

\section{Annonaceae}

Annona pickelii (Diels) H.Rainer

Guatteria schomburgkiana Mart.

Xylopia frutescens Aubl.

Xylopia laevigata (Mart.) R.E.Fr.

Apocynaceae

Blepharodon pictum (Vahl) W.D.Stevens

\section{HÁBITO COLETOR/N ${ }^{\circ}$}

Tre

Erv

Arv

Arv

Arv

Arv

Arv

Arv

Arv

Tre
Pereira, L.A. \& Chagas, E.C.O. 254

Pereira, L.A. 26

Pereira, L.A. 23

Pereira, L.A. \& Chagas, E.C.O. 102

Pereira, L.A. \& Chagas, E.C.O. 99

Pereira, L.A. \& Chagas, E.C.O. 265

Pereira, L.A. \& Chagas, E.C.O. 172

Pereira, L.A. 30

Pereira, L.A. \& Chagas, E.C.O. 264

Pereira, L.A. \& Chagas, E.C.O. 191 
Ditassa sp.

Hancornia speciosa Gomes

Himatanthus phagedaenicus (Mart.) Woodson

Mandevilla scabra (Hoffmanns. ex Roem. \& Schult.) K. Schum.

Mandevilla sp.

\section{Asteraceae}

Tilesia baccata (L.f.) Pruski

Struchium sparganophorum (L.) Kuntze

\section{Boraginaceae}

Cordia superba Cham.

Tournefortia candidula (Miers) Johnst.

\section{Bromeliaceae}

Hohenbergia sp.

\section{Burseraceae}

Protium heptaphyllum (Aubl.) Marchand

\section{Cactaceae}

Melocactus sp.

Cannabaceae

Trema micrantha (L.) Blume

\section{Celastraceae}

Maytenus erythroxylum Reissek

Chrysobalanaceae

Hirtella racemosa Lam.

\section{Clusiaceae}

Garcinia sp.

\section{Combretaceae}

Buchenavia tetraphylla (Aubl.) R.A.Howard

\section{Commelinaceae}

Commelina erecta L.

Convolvulaceae

Ipomoea quamoclit L.

Cucurbitaceae

Luffa cylindrica M. Roem.

Melothria fluminensis Gardner

Momordica charantia L.

\section{Cyperaceae}

Bulbostylis sp.

Cyperus laxus Lam.

Scleria bracteata Cav.

Scleria hirta Boeckeler

Scleria secans (L.) Urb.

Rhynchospora exaltata Kunth
Tre Pereira, L.A. \& Chagas, E.C.O. 260

Arv Pereira, L.A. \& Chagas, E.C.O. 158

Arv Pereira, L.A. \& Chagas, E.C.O. 69

Tre Pereira, L.A. \& Chagas, E.C.O. 150

Tre Pereira, L.A. \& Chagas, E.C.O. 261

Arb Pereira, L.A. 46

Erv Pereira, L.A. \& Chagas, E.C.O. 89

Arv Pereira, L.A. \& Chagas, E.C.O. 97

Tre Pereira, L.A. \& Chagas, E.C.O. 221

Erv Pereira, L.A. \& Chagas, E.C.O. 267

Arv Pereira, L.A. 50

Erv Pereira, L.A. \& Chagas, E.C.O. 268

Arv Pereira, L.A. \& Chagas, E.C.O. 81

Arv Pereira, L.A. 24

Arv Pereira, L.A. \& Chagas, E.C.O. 83

Arv Pereira, L.A. \& Chagas, E.C.O. 104

Arv Pereira, L.A. \& Chagas, E.C.O. 247

Erv Pereira, L.A. \& Vieira, L.A.F. 207

Tre $\quad$ Pereira, L.A. \& Chagas, E.C.O. 241

Tre Pereira, L.A. 47

Tre Pereira, L.A. \& Chagas, E.C.O. 187

Tre Pereira, L.A. \& Vieira, L.A.F. 209

Erv $\quad$ Pereira, L.A. \& Chagas, E.C.O. 248

Erv Pereira, L.A. \& Chagas, E.C.O. 142

Erv Pereira, L.A. \& Chagas, E.C.O. 146

Erv Pereira, L.A. \& Chagas, E.C.O. 188

Erv Pereira, L.A. \& Chagas, E.C.O. 147

Erv Pereira, L.A. \& Chagas, E.C.O. 189 


\section{Dilleniaceae}

Davilla kunthii A. St.-Hil.

Doliocarpus dentatus (Aubl.) Standl.

Tetracera breyniana Schltdl.

\section{Dioscoreaceae}

Dioscorea sp.

Elaeocarpaceae

Sloanea garckeana K.Schum.

\section{Eriocaulaceae}

Paepalanthus sp.

Erythroxylaceae

Erythroxylum sp.

Euphorbiaceae

Chaetocarpus myrsinites Baill.

Croton polyandrus Spreng.

Croton sellowii Baill.

Dalechampia sp.1

Dalechampia sp.2

Pogonophora schombugkiana Miers ex Benth.

Ricinus communis L.

Sapium sp.

Fabaceae

Abarema sp.

Andira sp.1

Andira sp.2

Apuleia leiocarpa (Vogel) J.F.Macbr.

Chamaecrista ramosa (Vogel) H.S.Irwin \& Barneby

Dioclea virgata (Rich.) Amshoff

Hymenaea courbaril L.

Inga thibaudiana DC.

Inga capitata Desv.

Inga sp.

Pterocarpus rohrii Vahl

Senna alata (L.) Roxb.

Senna sp.

Stylosanthes sp.

Heliconiaceae

Heliconia psittacorum L. f.

Hernandiaceae

Sparattanthelium botocudorum Mart.

Humiriaceae

Sacoglottis mattogrossensis Malme
Tre Pereira, L.A. 54

Tre Pereira, L.A. 53

Tre Pereira, L.A. \& Chagas, E.C.O. 100

Tre Pereira, L.A. \& Chagas, E.C.O. 240

Arv Moura, O.T. 1141

Erv Pereira, L.A. 27

Arb

Arv

Arv

Erv

Tre

Tre

Arv

Arb

Arv

Arv

Arv

Arv

Arv

Erv

Tre

Arv

Arv

Arv

Arv

Arv

Arb

Arv

Erv

Erv

Pereira, L.A. \& Vieira, L.A.F. 202

Arb

Pereira, L.A. \& Chagas, E.C.O. 174

Arv 


\begin{tabular}{|c|c|c|}
\hline \multicolumn{3}{|l|}{ Hypericaceae } \\
\hline Vismia guianensis (Aubl.) Choisy & Arb & Pereira, L.A. 25 \\
\hline \multicolumn{3}{|l|}{ Krameriaceae } \\
\hline Krameria tomentosa A.St.-Hil. & Arb & Pereira, L.A. \& Chagas, E.C.O. 246 \\
\hline \multicolumn{3}{|l|}{ Lamiaceae } \\
\hline Aegiphila sp. & Arb & Pereira, L.A. \& Chagas, E.C.O. 103 \\
\hline \multicolumn{3}{|l|}{ Lauraceae } \\
\hline Cassytha filiformis L. & Para & Pereira, L.A. \& Chagas, E.C.O. 92 \\
\hline Ocotea gardneri (Meisn.) Mez & Arv & Pereira, L.A. \& Chagas, E.C.O. 231 \\
\hline \multicolumn{3}{|l|}{ Lecythidaceae } \\
\hline Eschweilera ovata (Cambess.) Miers & Arv & Pereira, L.A. 52 \\
\hline \multicolumn{3}{|l|}{ Loranthaceae } \\
\hline Phoradendron sp. & Para & Pereira, L.A. \& Chagas, E.C.O. 223 \\
\hline Phthirusa pyrifolia (Kunth) Eichler & Para & Pereira, L.A. \& Chagas, E.C.O. 106 \\
\hline Psittacanthus dichroos (Mart.) Mart. & Para & Pereira, L.A. \& Chagas, E.C.O. 237 \\
\hline Struthanthus sp. & Para & Pereira, L.A. \& Chagas, E.C.O. 239 \\
\hline \multicolumn{3}{|l|}{ Lythraceae } \\
\hline Cuphea flava Spreng. & Erv & Pereira, L.A. \& Chagas, E.C.O. 232 \\
\hline \multicolumn{3}{|l|}{ Malpighiaceae } \\
\hline Byrsonima gardneriana A. Juss. & Arb & Pereira, L.A. 28 \\
\hline Byrsonima sericea DC. & Arv & Pereira, L.A. \& Chagas, E.C.O. 105 \\
\hline Stigmaphyllon rotundifolium A. Juss. & Tre & Pereira, L.A. \& Chagas, E.C.O. 171 \\
\hline \multicolumn{3}{|l|}{ Malvaceae } \\
\hline Eriotheca sp. & Arv & Pereira, L.A. \& Chagas, E.C.O. 253 \\
\hline Luehea ochrophylla Mart. & Arv & Pereira, L.A. \& Chagas, E.C.O. 143 \\
\hline Sida elata Macfad. & Erv & Pereira, L.A. \& Chagas, E.C.O. 179 \\
\hline Sida sp. & Erv & Pereira, L.A. \& Chagas, E.C.O. 90 \\
\hline \multicolumn{3}{|l|}{ Marantaceae } \\
\hline Monotagma plurispicatum (Körn.) K. Schum. & Erv & Pereira, L.A. \& Chagas, E.C.O. 68 \\
\hline \multicolumn{3}{|l|}{ Melastomataceae } \\
\hline Miconia albicans (Sw.) Triana & Arv & Pereira, L.A. \& Chagas, E.C.O. 74 \\
\hline \multicolumn{3}{|l|}{ Myrsinaceae } \\
\hline Myrsine sp. & Arb & Pereira, L.A. \& Chagas, E.C.O. 224 \\
\hline \multicolumn{3}{|l|}{ Myrtaceae } \\
\hline Eugenia azurensis O.Berg & Arv & Pereira, L.A. \& Chagas, E.C.O. 16o \\
\hline Eugenia candolleana DC. & Arb & Pereira, L.A. \& Chagas, E.C.O. 178 \\
\hline Eugenia hirta O. Berg & Arb & Pereira, L.A. \& Chagas, E.C.O. 159 \\
\hline Eugenia punicifolia (Kunth) DC. & Arb & Pereira, L.A. \& Chagas, E.C.O. 88 \\
\hline Myrcia bergiana O.Berg. & Arv & Pereira, L.A. \& Chagas, E.C.O. 169 \\
\hline Myrcia guianensis (Aubl.) DC. & Arv & Pereira, L.A. \& Chagas, E.C.O. 185 \\
\hline Myrcia sylvatica (G. Mey.) DC. & Arv & Pereira, L.A. \& Chagas, E.C.O. 176 \\
\hline Myrciaria sp. & Arb & Pereira, L.A. \& Chagas, E.C.O. 244 \\
\hline
\end{tabular}


Nyctaginaceae

Guapira pernambucensis (Casar.) Lundell

Ochnaceae

Ouratea hexasperma (A.St.-Hil.) Baill.

Onagraceae

Ludwigia octovalvis (Jacq.) P.H. Raven

Orchidaceae

Epidendrum cinnabarinum Salzm.

Oeceoclades maculata (Lindl.) Lindl.

Passifloraceae

Passiflora cincinata Mast.

Passiflora galbana Mast.

Pcramniaceae

Picramnia sp.

Piperaceae

Piper tuberculatum Jacq.

Plumbaginaceae

Plumbago scandens L.

Poaceae

Digitaria sp.

Ichnanthus sp.1

Ichnanthus sp.2

Lasiacis ligulata Hitchc. \& Chase

Panicum asperifolium (Desv.) Hitchc.

Panicum pilosum Sw.

Panicum sp.

Paspalum sp.

Polygalaceae

Bredemeyera laurifolia Klotzsch

Polygala sp.1

Polygala sp.2

Polygala sp.3

Polygonaceae

Coccoloba alnifolia Casar.

Coccolaba laevis Casar.

Rubiaceae

Alseis pickelii Pilg. \& Schmale

Borreria verticillata (L.) G.Mey.

Chiococca alba (L.) Hitchc.

Cordiera myrciifolia (K.Schum.) C.H.Perss. \& Delprete

Denscantia cymosa (Spreng.) E.L.Cabral \& Bacigalupo
Arb Pereira, L.A. \& Chagas, E.C.O. 155

Arv Pereira, L.A. \& Chagas, E.C.O. 185

Erv Pereira, L.A. 45

Erv Pereira, L.A. \& Chagas, E.C.O. 255

Erv Pereira, L.A. \& Chagas, E.C.O. 238

Tre Pereira, L.A. \& Chagas, E.C.O. 183

Tre Pereira, L.A. \& Chagas, E.C.O. 168

Arv Pereira, L.A. \& Chagas, E.C.O. 228

Arb Pereira, L.A. 48

Erv Pereira, L.A. 32

Erv Pereira, L.A. \& Chagas, E.C.O. 252

Erv Pereira, L.A. \& Chagas, E.C.O. 250

Erv Pereira, L.A. \& Chagas, E.C.O. 251

Erv Pereira, L.A. 33

Erv Pereira, L.A. \& Chagas, E.C.O. 144

Erv Pereira, L.A. \& Chagas, E.C.O. 190

Erv Pereira, L.A. \& Chagas, E.C.O. 153

Erv Pereira, L.A. \& Chagas, E.C.O. 154

Erv Pereira, L.A. \& Chagas, E.C.O. 184

Erv Pereira, L.A. \& Chagas, E.C.O. 107

Erv Pereira, L.A. \& Chagas, E.C.O. 222

Erv Pereira, L.A. \& Chagas, E.C.O. 227

Arv Pereira, L.A. \& Chagas, E.C.O. 82

Tre Pereira, L.A. \& Chagas, E.C.O. 219

Arb Moura, O.T. 1089

Erv $\quad$ Pereira, L.A. \& Vieira, L.A.F. 206

Arb Pereira, L.A. \& Chagas, E.C.O. 245

Arb Pereira, L.A. \& Chagas, E.C.O. 87

Arb Pereira, L.A. \& Chagas, E.C.O. 257 
Guettarda platypoda DC.

Mitracarpus sp.

Palicourea crocea (Sw.) Roem. \& Schult.

Posoqueria longiflora Aubl.

Psychotria barbiflora DC.

Salzmannia nitida DC.

Tocoyena sellowiana (Cham. \& Schltdl.) K.Schum.

\section{Salicaceae}

Casearia javitensis Kunth

Sapindaceae

Allophylus laevigatus Radlk.

Serjania salzmanniana Schltr.

Sapotaceae

Manilkara salzmannii (A. DC.) H.J.Lam.

Pouteria sp.

\section{Schoepfiaceae}

Schoepfia brasiliensis A.DC.

Smilacaceae

Smilax sp.1

Smilax sp.2

Solanaceae

Physalis angulata L.

Solanum paludosum Moric.

Solanum torvum Sw.

Solanum sp.

Trigoniaceae

Trigonia nivea Cambess.

Urticaceae

Cecropia sp.

Verbenaceae

Lippia sp.

Vitaceae

Cissus erosa Rich.
Arv Pereira, L.A. 29

Erv Pereira, L.A. \& Chagas, E.C.O. 256

Arb Pereira, L.A. \& Vieira, L.A.F. 204

Arv Pereira, L.A. \& Vieira, L.A.F. 210

Erv Pereira, L.A. \& Vieira, L.A.F. 205

Arb Pereira, L.A. \& Chagas, E.C.O. 186

Arv Pereira, L.A. \& Chagas, E.C.O. 181

Arv Pereira, L.A. \& Chagas, E.C.O. 78

Arv Pereira, L.A. 31

Tre Pereira, L.A. \& Chagas, E.C.O. 95

Arv Pereira, L.A. \& Chagas, E.C.O. 156

Arv Pereira, L.A. \& Chagas, E.C.O. 91

Arv Pereira, L.A. \& Chagas, E.C.O. 226

Tre Pereira, L.A. \& Chagas, E.C.O. 166

Tre Pereira, L.A. \& Chagas, E.C.O. 234

Erv Pereira, L.A. \& Chagas, E.C.O. 259

Arb Pereira, L.A. \& Chagas, E.C.O. 71

Arb Pereira, L.A. \& Chagas, E.C.O. 73

Arb Pereira, L.A. \& Chagas, E.C.O. 72

Tre Pereira, L.A. \& Chagas, E.C.O. 96

Arv Pereira, L.A. \& Chagas, E.C.O. 75

Erv Pereira, L.A. \& Chagas, E.C.O. 77

Tre Pereira, L.A. \& Chagas, E.C.O. 145

Dois gêneros e cinco espécies são exclusivos deste trabalho quando comparados com os demais levantamentos utilizados na análise de similaridade florística, Melocactus Link \& Otto, endêmico do Brasil e Garcinia L. que é aqui referido pela primeira vez para o Estado da Paraíba (SOMNER et al. 2013). Entre as espécies destacam-se Psittacanthus dichroos (Mart.) Mart. e Denscantia cymosa (Spreng.) E.L. Cabral \& Bacigalupo, endêmicas do Brasil e Struchium sparganophorum (L.) Kuntze que é referida pela primeira vez para o Estado da Paraíba (SOMNER et al. 2013).

Do total de espécies 54 (36\%) são árvores, 39 (26\%) ervas, 27 (17\%) arbustos, 26 (18\%) trepadeiras e $5(3 \%)$ parasitas. O predomínio de espécies arbóreas caracteriza 
uma vegetação de fisionomia florestal, fato também observado em três dos estudos utilizados na comparação florística (AMAZONAS, 2011; BARBOSA, 2008; BARBOSA et al. 2009). Entretanto a presença de Byrsonima sericea DC., Krameria tomentosa A.St.-Hil. e Hancornia speciosa Gomes, normalmente coletadas em áreas savanoides de solos arenosos, evidenciam a presença de outras fitofisionomias no remanescente do Rio Cabelo.

O remanescente de mata ciliar do Rio Jaguaribe foi a única área com predomínio de espécies herbáceas (41\%) em detrimento de arbóreas, característica associada à perturbação ambiental (FREITAS, 2011). O Rio Jaguaribe vem sofrendo diversas intervenções humanas desde a década de 1940, onde parte do seu leito foi aterrado e seu curso desviado (JOÃO PESSOA, 2007). Estes fatores em conjunto são indicadores da acentuada ação antrópica nesse remanescente.

De acordo com GIBBS e LEITÃO-FILHO (1978) as espécies do gênero Inga juntamente com Tapirira guianensis e Protium heptaphyllum são típicas de formações ciliares, principalmente devido à capacidade de sobreviverem em solos inundáveis. Apesar disso as espécies supracitadas não são exclusivas dessas matas, sendo encontradas em vários outros levantamentos realizados em formações não ciliares no estado (BARBOSA et al. 2011; DIONÍSIO, 2002; PONTES, 2000).

Segundo IVANAUSKAS et al. (1997) a composição florística das matas ciliares é fortemente influenciada pela vegetação do interflúvio, o que torna necessário um conhecimento acerca do mosaico vegetacional em que estão inseridas. Assim como observado por outros autores, que estudam a vegetação ciliar no Brasil (RODRIGUES e NAVE, 200o), o remanescente de mata ciliar do Rio Cabelo não apresenta uma "flora específica", mas sim um misto de espécies tolerantes à dinâmica hidrológica, amplamente distribuídas na Floresta Atlântica Nordestina e algumas com distribuição disjunta com a Floresta Amazônica.

Na comparação florística, apresentada na tabela 2, a área que obteve maior índice de similaridade com o presente estudo foi o remanescente da bacia hidrográfica do rio Timbó (26.4\%), seguida da falésia do Cabo Branco (22.9\%), mata do Buraquinho (20.1\%) e da bacia hidrográfica do rio Jaguaribe (16.6\%).

Tabela 2. Comparação florística entre a área de estudo e outras áreas da região metropolitana de João Pessoa: 1. Mata ciliar da bacia hidrográfica do Rio Timbó

(Amazonas, 2006); 2. Mata ciliar da bacia hidrográfica do rio Jaguaribe (Freita, 2011) 3.

Mata do Buraquinho (Barbosa, 2008); 4. Falésia do Cabo Branco (Barbosa et al., 2009).

\begin{tabular}{|l|c|c|c|c|}
\hline Família/espécie & $\mathbf{1}$ & $\mathbf{2}$ & $\mathbf{3}$ & $\mathbf{4}$ \\
\hline ACANTHACEAE & & & & \\
\hline Ruellia paniculata L. & & & $\mathbf{X}$ & $\mathbf{X}$ \\
\hline ANACARDIACEAE & & & & \\
\hline Anacardium occidentale L. & $\mathbf{X}$ & $\mathbf{X}$ & & $\mathbf{X}$ \\
\hline Tapirira guianensis Aubl. & $\mathbf{X}$ & $\mathbf{X}$ & $\mathbf{X}$ & $\mathbf{X}$ \\
\hline
\end{tabular}




\begin{tabular}{|c|c|c|c|c|}
\hline Família/espécie & 1 & 2 & 3 & 4 \\
\hline Thyrsodium spruceanum Benth. & $\mathbf{X}$ & & $\mathrm{X}$ & $\mathbf{X}$ \\
\hline \multicolumn{5}{|l|}{ ANNONACEAE } \\
\hline Annona pickelii (Diels) H.Rainer & $\mathbf{X}$ & & $\mathbf{X}$ & \\
\hline Guatteria schomburgkiana Mart. & $\mathbf{X}$ & & $\mathbf{X}$ & $\mathbf{X}$ \\
\hline Xylopia frutescens Aubl. & & & $\mathbf{X}$ & \\
\hline \multicolumn{5}{|l|}{ Xylopia laevigata (Mart.) R.E.Fr. } \\
\hline \multicolumn{5}{|l|}{ APOCYNACEAE } \\
\hline Blepharodon pictum (Vahl) W.D.Stevens & & & $\mathbf{X}$ & \\
\hline Hancornia speciosa Gomes & & & & $\mathbf{X}$ \\
\hline Himatanthus phagedaenicus (Mart.) Woodson & $\mathrm{X}$ & $\mathrm{X}$ & $\mathrm{X}$ & $\mathbf{X}$ \\
\hline Mandevilla scabra (Hoffmanns. ex Roem. \& Schult.) K. Schum. & $\mathbf{X}$ & & $\mathbf{X}$ & \\
\hline \multicolumn{5}{|l|}{ ASTERACEAE } \\
\hline \multicolumn{5}{|l|}{ Struchium sparganophorum (L.) Kuntze } \\
\hline Tilesia baccata (L.f.) Pruski & $\mathbf{X}$ & & $\mathbf{X}$ & $\mathbf{X}$ \\
\hline \multicolumn{5}{|l|}{ BORAGINACEAE } \\
\hline Cordia superba Cham. & & & $\mathbf{X}$ & \\
\hline Tournefortia candidula (Miers) Johnst. & & $\mathbf{X}$ & $\mathbf{X}$ & $\mathbf{X}$ \\
\hline \multicolumn{5}{|l|}{ BURSERACEAE } \\
\hline Protium heptaphyllum (Aubl.) Marchand & $\mathbf{X}$ & $\mathbf{X}$ & $\mathbf{X}$ & $\mathbf{X}$ \\
\hline \multicolumn{5}{|l|}{ CANNABACEAE } \\
\hline Trema micrantha (L.) Blume & $\mathbf{X}$ & $\mathbf{X}$ & $\mathbf{X}$ & $\mathbf{X}$ \\
\hline \multicolumn{5}{|l|}{ CELASTRACEAE } \\
\hline Maytenus erythroxyla Reissek & $\mathbf{X}$ & & $\mathbf{X}$ & $\mathbf{X}$ \\
\hline \multicolumn{5}{|l|}{ CHRYSOBALANACEAE } \\
\hline Hirtella racemosa Lam. & $\mathbf{X}$ & $\mathbf{X}$ & $\mathrm{X}$ & $\mathbf{X}$ \\
\hline \multicolumn{5}{|l|}{ COMBRETACEAE } \\
\hline Buchenavia tetraphylla (Aubl.) R.A.Howard & $\mathbf{X}$ & & $\mathbf{X}$ & \\
\hline \multicolumn{5}{|l|}{ COMMELINACEAE } \\
\hline \multicolumn{5}{|l|}{ Commelina erecta $\mathrm{L}$. } \\
\hline \multicolumn{5}{|l|}{ CONVOLVULACEAE } \\
\hline \multicolumn{5}{|l|}{ Ipomoea quamoclit L. } \\
\hline \multicolumn{5}{|l|}{ CUCURBITACEAE } \\
\hline \multicolumn{5}{|l|}{ Luffa cylindrica M. Roem. } \\
\hline \multicolumn{5}{|l|}{ Melothria fluminensis Gardner } \\
\hline Momordica charantia L. & $\mathbf{X}$ & & & $\mathbf{X}$ \\
\hline
\end{tabular}




\begin{tabular}{|c|c|c|c|c|}
\hline Família/espécie & 1 & 2 & 3 & 4 \\
\hline \multicolumn{5}{|l|}{ CYPERACEAE } \\
\hline Cyperus laxus Lam. & & & $\mathbf{X}$ & \\
\hline Scleria bracteata Cav. & $\mathbf{X}$ & $\mathbf{X}$ & $\mathbf{X}$ & \\
\hline \multicolumn{5}{|l|}{ Scleria hirta Boeckeler } \\
\hline \multicolumn{5}{|l|}{ Scleria secans (L.) Urb. } \\
\hline \multicolumn{5}{|l|}{ Rhynchospora exaltata Kunth } \\
\hline \multicolumn{5}{|l|}{ DILLENIACEAE } \\
\hline Davilla kunthii A. St.-Hil. & & & $\mathbf{X}$ & \\
\hline \multicolumn{5}{|l|}{ Doliocarpus dentatus (Aubl.) Standl. } \\
\hline Tetracera breyniana Schltdl. & $\mathbf{X}$ & $\mathbf{X}$ & $\mathbf{X}$ & $\mathbf{X}$ \\
\hline \multicolumn{5}{|l|}{ ELAEOCARPACEAE } \\
\hline Sloanea garckeana K.Schum. & & & $\mathbf{X}$ & \\
\hline \multicolumn{5}{|l|}{ EUPHORBIACEAE } \\
\hline Chaetocarpus myrsinites Baill. & $\mathbf{X}$ & & $\mathbf{X}$ & $\mathbf{X}$ \\
\hline Croton polyandrus Spreng. & & & $\mathbf{X}$ & \\
\hline Croton sellowii Baill. & $\mathbf{X}$ & & & \\
\hline Pogonophora schombugkiana Miers ex Benth. & $\mathbf{X}$ & & $\mathbf{X}$ & \\
\hline Ricinus communis L. & $\mathbf{X}$ & & & \\
\hline \multicolumn{5}{|l|}{ FABACEAE } \\
\hline Apuleia leiocarpa (Vogel) J.F.Macbr. & & $\mathbf{X}$ & $\mathbf{X}$ & $\mathbf{X}$ \\
\hline Chamaecrista ramosa (Vogel) H.S.Irwin \& Barneby & & & $\mathbf{X}$ & \\
\hline Dioclea virgata (Rich.) Amshoff & $\mathbf{X}$ & $\mathbf{X}$ & $\mathbf{X}$ & $\mathbf{X}$ \\
\hline Hymenaea courbaril L. & & $\mathbf{X}$ & & $\mathbf{X}$ \\
\hline Inga capitata Desv. & $\mathrm{X}$ & & $\mathrm{X}$ & \\
\hline Inga thibaudiana DC. & & & $\mathrm{X}$ & \\
\hline Pterocarpus rohrii Vahl & $\mathrm{X}$ & & $\mathbf{X}$ & \\
\hline Senna alata (L.) Roxb. & & $\mathbf{X}$ & & \\
\hline \multicolumn{5}{|l|}{ HELICONIACEAE } \\
\hline Heliconia psittacorum L. f. & $\mathbf{X}$ & $\mathbf{X}$ & $\mathbf{X}$ & $\mathbf{X}$ \\
\hline \multicolumn{5}{|l|}{ HERNANDIACEAE } \\
\hline Sparattanthelium botocudorum Mart. & $\mathrm{X}$ & $\mathrm{X}$ & $\mathrm{X}$ & $\mathbf{X}$ \\
\hline \multicolumn{5}{|l|}{ HUMIRIACEAE } \\
\hline Sacoglottis mattogrossensis Malme & & & $\mathrm{X}$ & $\mathrm{X}$ \\
\hline \multicolumn{5}{|l|}{ HYPERICACEAE } \\
\hline Vismia guianensis (Aubl.) Choisy & $\mathrm{X}$ & $\mathrm{X}$ & & $\mathrm{X}$ \\
\hline
\end{tabular}




\begin{tabular}{|c|c|c|c|c|}
\hline Família/espécie & 1 & 2 & 3 & 4 \\
\hline \multicolumn{5}{|l|}{ KRAMERIACEAE } \\
\hline \multicolumn{5}{|l|}{ Krameria tomentosa A.St.-Hil. } \\
\hline \multicolumn{5}{|l|}{ LAURACEAE } \\
\hline Cassytha filiformis L. & & $\mathbf{X}$ & & $\mathbf{X}$ \\
\hline Ocotea gardneri (Meisn.) Mez & & & $\mathbf{X}$ & \\
\hline \multicolumn{5}{|l|}{ LECYTHIDACEAE } \\
\hline Eschweilera ovata (Cambess.) Miers & $\mathbf{X}$ & & $\mathbf{X}$ & $\mathbf{X}$ \\
\hline \multicolumn{5}{|l|}{ LORANTHACEAE } \\
\hline \multicolumn{5}{|l|}{ Phthirusa pyrifolia (Kunth) Eichler } \\
\hline \multicolumn{5}{|l|}{ Psittacanthus dichroos (Mart.) Mart. } \\
\hline \multicolumn{5}{|l|}{ LYTHRACEAE } \\
\hline Cuphea flava Spreng. & & & & $\mathbf{X}$ \\
\hline \multicolumn{5}{|l|}{ MALPIGHIACEAE } \\
\hline Byrsonima gardneriana A. Juss. & $\mathbf{X}$ & & & $\mathbf{X}$ \\
\hline Byrsonima sericea DC. & $\mathbf{X}$ & & $\mathbf{X}$ & $\mathbf{X}$ \\
\hline Stigmaphyllon rotundifolium A. Juss. & $\mathbf{X}$ & $\mathbf{X}$ & & \\
\hline \multicolumn{5}{|l|}{ MALVACEAE } \\
\hline Luehea ochrophylla Mart. & $\mathbf{X}$ & & $\mathbf{X}$ & $\mathbf{X}$ \\
\hline \multicolumn{5}{|l|}{ Sida elata Macfad. } \\
\hline \multicolumn{5}{|l|}{ MARANTACEAE } \\
\hline Monotagma plurispicatum (Körn.) K. Schum. & & & $\mathbf{X}$ & \\
\hline \multicolumn{5}{|l|}{ MELASTOMATACEAE } \\
\hline Miconia albicans (Sw.) Triana & $\mathbf{X}$ & $\mathbf{X}$ & $\mathbf{X}$ & $\mathbf{X}$ \\
\hline \multicolumn{5}{|l|}{ MYRTACEAE } \\
\hline \multicolumn{5}{|l|}{ Eugenia azurensis O.Berg } \\
\hline \multicolumn{5}{|l|}{ Eugenia candolleana DC. } \\
\hline Eugenia hirta O. Berg & & $\mathbf{X}$ & & $\mathbf{X}$ \\
\hline Eugenia punicifolia (Kunth) DC. & $\mathbf{X}$ & $\mathbf{X}$ & $\mathbf{X}$ & $\mathbf{X}$ \\
\hline Myrcia bergiana O.Berg & & & $\mathbf{X}$ & \\
\hline Myrcia guianensis (Aubl.) DC. & & & & $\mathbf{X}$ \\
\hline Myrcia sylvatica (G. Mey.) DC. & $\mathbf{X}$ & & $\mathbf{X}$ & $\mathbf{X}$ \\
\hline \multicolumn{5}{|l|}{ NYCTAGINACEAE } \\
\hline Guapira pernambucensis (Casar.) Lundell & & & & $\mathbf{X}$ \\
\hline \multicolumn{5}{|l|}{ OCHNACEAE } \\
\hline Ouratea hexasperma (A.St.-Hil.) Baill. & & & $\mathbf{X}$ & $\mathbf{X}$ \\
\hline
\end{tabular}




\begin{tabular}{|c|c|c|c|c|}
\hline Família/espécie & 1 & 2 & 3 & 4 \\
\hline \multicolumn{5}{|l|}{ ONAGRACEAE } \\
\hline \multicolumn{5}{|l|}{ Ludwigia octovalvis (Jacq.) P.H. Raven } \\
\hline \multicolumn{5}{|l|}{ ORCHIDACEAE } \\
\hline \multicolumn{5}{|l|}{ Epidendrum cinnabarinum Salzm. } \\
\hline Oeceoclades maculata (Lindl.) Lindl. & $\mathbf{X}$ & & & $\mathbf{X}$ \\
\hline \multicolumn{5}{|l|}{ PASSIFLORACEAE } \\
\hline \multicolumn{5}{|l|}{ Passiflora cincinata Mast. } \\
\hline \multicolumn{5}{|l|}{ Passiflora galbana Mast. } \\
\hline \multicolumn{5}{|l|}{ PIPERACEAE } \\
\hline Piper tuberculatum Jacq. & & & & $\mathbf{X}$ \\
\hline \multicolumn{5}{|l|}{ PLUMBAGINACEAE } \\
\hline Plumbago scandens L. & & $\mathbf{X}$ & & $\mathbf{X}$ \\
\hline \multicolumn{5}{|l|}{ POACEAE } \\
\hline \multicolumn{5}{|l|}{ Lasiacis ligulata Hitchc. \& Chase } \\
\hline Panicum asperifolium (Desv.) Hitchc. & & $\mathbf{x}$ & & \\
\hline Panicum pilosum $\mathrm{Sw}$. & & $\mathbf{X}$ & $\mathbf{X}$ & \\
\hline \multicolumn{5}{|l|}{ POLYGALACEAE } \\
\hline Bredemeyera laurifolia Klotzsch & $\mathbf{X}$ & & $\mathbf{X}$ & \\
\hline \multicolumn{5}{|l|}{ POLYGONACEAE } \\
\hline Coccoloba alnifolia Casar. & & $\mathrm{X}$ & & $\mathbf{X}$ \\
\hline Coccolaba laevis Casar. & & & & $\mathbf{X}$ \\
\hline \multicolumn{5}{|l|}{ RUBIACEAE } \\
\hline Alseis pickelii Pilg. \& Schmale & & & $\mathbf{X}$ & \\
\hline Borreria verticillata (L.) G.Mey. & & $\mathbf{X}$ & $\mathbf{X}$ & $\mathbf{X}$ \\
\hline Chiococca alba (L.) Hitchc. & $\mathbf{X}$ & $\mathbf{X}$ & $\mathbf{X}$ & $\mathbf{X}$ \\
\hline \multicolumn{5}{|l|}{ Cordiera myrciifolia (K.Schum.) C.H.Perss. \& Delprete } \\
\hline \multicolumn{5}{|l|}{ Denscantia cymosa (Spreng.) E.L.Cabral \& Bacigalupo } \\
\hline Guettarda platypoda DC. & $\mathbf{X}$ & & $\mathbf{X}$ & $\mathbf{X}$ \\
\hline Palicourea crocea (Sw.) Roem. \& Schult. & $\mathbf{X}$ & & $\mathbf{X}$ & \\
\hline Posoqueria longiflora Aubl. & & & $\mathbf{X}$ & \\
\hline Psychotria barbiflora DC. & $\mathbf{X}$ & & $\mathbf{X}$ & \\
\hline Salzmannia nitida DC. & $\mathbf{X}$ & & $\mathbf{X}$ & \\
\hline Tocoyena sellowiana (Cham. \& Schltdl.) K.Schum. & $\mathbf{X}$ & & $\mathbf{X}$ & $\mathbf{X}$ \\
\hline \multicolumn{5}{|l|}{ SALICACEAE } \\
\hline Casearia javitensis Kunth & & & & $\mathbf{X}$ \\
\hline
\end{tabular}




\begin{tabular}{|l|c|c|c|c|}
\hline Família/espécie & $\mathbf{1}$ & $\mathbf{2}$ & $\mathbf{3}$ & $\mathbf{4}$ \\
\hline SAPINDACEAE & & & & \\
\hline Allophylus laevigatus Radlk. & $\mathrm{X}$ & $\mathrm{X}$ & $\mathrm{X}$ & $\mathrm{X}$ \\
\hline Serjania salzmanniana Schltr. & $\mathrm{X}$ & & $\mathrm{X}$ & $\mathrm{X}$ \\
\hline SAPOTACEAE & & & & \\
\hline Manilkara salzmannii (A. DC.) H.J.Lam. & & & $\mathrm{X}$ & $\mathrm{X}$ \\
\hline SCHOEPFIACEAE & & & & \\
\hline Schoepfia brasiliensis A.DC. & $\mathrm{X}$ & & & \\
\hline SOLANACEAE & & & & \\
\hline Physalis angulata L. & & & & \\
\hline Solanum paludosum Moric. & & $\mathrm{X}$ & $\mathrm{X}$ & $\mathrm{X}$ \\
\hline Solanum torvum Sw. & & & & \\
\hline TRIGONIACEAE & & & & \\
\hline Trigonia nivea Cambess. & $\mathrm{X}$ & & $\mathrm{X}$ & $\mathrm{X}$ \\
\hline VITACEAE & & & & \\
\hline Cissus erosa Rich. & $\mathrm{X}$ & & $\mathrm{X}$ & $\mathrm{X}$ \\
\hline Número total de espécies identificadas & $\mathbf{1 1 7}$ & $\mathbf{9 5}$ & $\mathbf{2 5 6}$ & $\mathbf{1 7 1}$ \\
\hline Número de espécies em comum com este trabalho & $\mathbf{4 7}$ & $\mathbf{2 9}$ & $\mathbf{6 1}$ & $\mathbf{5 2}$ \\
\hline IS (\%) & $\mathbf{2 6 . 4}$ & $\mathbf{1 6 . 6}$ & $\mathbf{2 0 . 1}$ & $\mathbf{2 2 . 9}$ \\
\hline
\end{tabular}

Os maiores índices de similaridade foram observados entre as áreas mais próximas espacialmente. A mata ciliar do Rio Cabelo quando comparada com a mata da Falésia do Cabo Branco obteve maior similaridade (22.9\%) do que quando comparada com a mata ciliar do Rio Jaguaribe (16.6\%), indicando que a distância geográfica pode ter influenciado nesses índices, como observado por RIBEIROFILHO et al. (2009). Vale ressaltar, porém, que a mata ciliar do Rio Jaguaribe, de todas as analisadas é aquela que se encontra mais antropizada.

Para as áreas ciliares, os dados obtidos corroboram estudos nessas formações, onde o número de espécies é bastante elevado e os valores de similaridade muito baixos (DURIGAN e LEITÃO-FILHO, 1995; MEGURO et al. 1996; SILVA JÚNIOR et al. 1998). Estes autores apontam a heterogeneidade ambiental como principal geradora dessas particularidades florísticas.

Os dados obtidos com este trabalho indicam que a mata ciliar da bacia hidrográfica do Rio Cabelo é bastante heterogênea floristicamente, sendo necessários mais estudos florísticos e ecológicos para que se possa entender sua estrutura e dinâmica, visando futuras ações de manejo e recuperação dessa formação. 


\section{AGRADECIMENTOS}

Agradecemos à Secretaria do Meio Ambiente da Prefeitura Municipal de João Pessoa pelo apoio técnico-operacional durante as coletas e ao $\mathrm{CNPq}$ pela bolsa de iniciação científica concedida ao primeiro autor.

\section{REFERENCIAS}

AMAZONAS, N. T. e BARBOSA, M. R. V. 2011 - Levantamento Florístico das Angiospermas em um remanescente de Floresta Atlântica Estacional na Microbacia Hidrográfica do Rio Timbó, João Pessoa, PB. Revista Nordestina de Biologia 2o(2): 6778.

ANGIOSPERM PHYLOGENY GROUP. 2009 - An update of the Angiosperm Phylogeny Group classification for the orders and families of flowering plants: APG III. Botanical Journal of the Linnean Society of London 161: 105-121.

BARBOSA, M.R.V. 2008 - Floristic Composition of a Remnant of Atlantic Coastal Forest in João Pessoa, Paraíba, Brazil. Memoirs of the New York Botanical Garden 100: 439-457.

BARBOSA, M.R.V.; LIMA, R.B.; LIMA, J.R.; LIMA, I.B.; GADELHA NETO, P.C.; ALMEIDA, A.; FREITAS, G.B. e MENEZES, C. 2009 - Estudo do Meio Biótico na Área de Influência da Erosão do Cabo Branco e Projeto de Regeneração da Praia do Cabo Branco, no Município de João Pessoa/PB. Relatório Técnico Final de Vegetação. João Pessoa.

BARBOSA, M.R.V. ; THOMAS, W. W. ; PAULA-ZÁRATE, E. L. ; LIMA, R. B. ; AGRA, M.F. ; LIMA, I.B. ; PESSOA, M.C.R. ; LOURENÇO, A.R. ; DELGADO JUNIOR, G.C. ; PONTES, R.A.S. ; CHAGAS, E.C.O. ; VIANA, J.L. ; GADELHA NETO, P.C. ; ARAUJO, C.M.L.R. ; ARAUJO, A.A.M. ; FREITAS, G.B. ; LIMA, J.R. ; SILVA, F.O. ; VIEIRA, L.A.F. ; PEREIRA, L. A. ; COSTA, R.M.T. ; DURÉ, R.C. e SÁ, M.G.V. 2011 - Checklist of the Vascular Plants of the Guaribas Biological Reserve, Paraíba, Brazil. Revista Nordestina de Biologia 20: 79-106.

DIONÍSIO, G.O. 2002 - Florística e fitossociologia do estrato arbóreo e arbustivo da RPPN Fazenda Pacatuba, Sapé/PB. Monografia de Bacharelado. João Pessoa, Universidade Federal da Paraíba. 52p.

DURIGAN, G. e LEITÃO FILHO, H.F. 1995 - Florística e Fitossociologia de Matas Ciliares do Oeste Paulista. Revta. Inst. Flor. São Paulo 7 (2): 197-239. 
FERNANDES, J. et al. 2010 - Estudo do processo de urbanização nas bacias hidrográficas dos rios Cabelo e Jacarapé, localizadas em João Pessoa - PB. Sistema de Gerenciamento de Conferências (OCS), V CONNEPI.

FORZZA, et al. 2010 - Introdução. In: Lista de Espécies da Flora do Brasil. Jardim Botânico do Rio de Janeiro (http://floradobrasil.jbrj.gov.br/).

FREITAS, G.B. 2011 - Composição Florística de Remanescentes de Mata Ciliar do Longo Alto e Médio Curso do Rio Jaguaribe, João Pessoa, PB. Dissertação de Mestrado. Programa Regional de Pós-Graduação em Desenvolvimento e Meio Ambiente, Universidade Federal da Paraíba. João Pessoa.

GIBBS, P.E. e LEITÃO-FILHO, H.F. 1978 - Floristic composition of an area of gallery forest near Mogi Guaçu, State of São Paulo, S.E. Brazil. Revista Brasileira de Botânica 1:151-156.

HINKEL, R. 2003 - Vegetação Ripária: Funções e Ecologia. In: Anais do I Seminário de Hidrologia Florestal: Zonas Ripárias. Programa de Pós-Graduação em Engenharia Ambiental/UFSC. p. 49-63.

IVANAUSKAS, N.M.; RODRIGUES, R.R. e NAVE, A.G. 1997 - Aspectos ecológicos de um trecho de floresta de brejo em Itatinga, SP: florística, fitossociologia e seletividade de espécies. Revista brasileira de Botânica 20(2): 139-150.

JOÃO PESSOA. 2007 - PLANO DE TRABALHO TECNICO SOCIAL (PTTS) - PAC do Alto Jaguaribe. João Pessoa - PB. Prefeitura Municipal de João Pessoa.

LEITE, E.P.F. 2005 - Caracterização hidrológica e de atributos físico - hídricos do solo dos solos da bacia hidrográfica do rio do Cabelo, utilizando sistemas computacionais livres. Tese de Doutorado. Universidade Federal de Campina Grande.

LIMA, W.P. e ZAKIA, M.J.B. 2000 - Hidrologia de matas ciliares. In: Rodrigues,R.R.; H.F. Leitão Filho (ed). Matas ciliares conservação e recuperação, São Paulo: EDUSP, FAPESP, p. 33-44.

LINDNER, E.A. 2003 - A legislação ambiental e as Áreas Ripárias. In: Anais do I Seminário de Hidrologia Florestal: Zonas Ripárias. Programa de Pós-Graduação em Engenharia Ambiental/UFSC. p. 49-63.

MEGURO, M.; PIRANI, J.R.; MELO-SILVA, R. e GIULIETTI, A.M. 1996 - Caracterização Florística e Estrututal de Matas Ripárias e Campões de Altitude da Serra do Cipó, Minas Gerais. Boletim de Botânica da Universidade de São Paulo 15: 13-29. 
MORI, S. A. et al. 1989 - Manual de manejo do herbário fanerogâmico. Ilhéus: Ed. Centro de Pesquisa do Cacau, 2 $2^{-\underline{a}}$ ed., 104 p.

MUELlER-DOMBOIS, D. e H. ELLENBERG. 1974 - Aims and Methods of Vegetation Ecology. Wiley, New York. 547 p.

OLIVEIRA, F. B. 2001 - Degradação do meio físico e implicações ambientais na Bacia do Rio Jaguaribe - João Pessoa. Dissertação de Mestrado. Universidade Federal de Pernambuco.

OLIVEIRA-FILHO, A. T.; RATTER, J. A. e SHEPHERED, G. J. 1990 - Floristic Composition and Community Structure of a Central Brazilian Gallery Forest. Flora 184: 103-107.

PONTES, A.F. 2000 - Levantamento florístico da Mata da AMEM, Cabedelo, Paraíba - Brasil. Monografia de Graduação. Universidade Federal da Paraíba.

RIBEIRO-FILHO, A. A.; FUNCH, L. S. e RODAL, M. J. N. 2009 - Composição Florística da Floresta Ciliar do Rio Mandassaia, Parque Nacional da Chapada Diamantina, Bahia, Brasil. Rodriguésia 6o (2): 265-276.

RODRIGUES, R.R. e NAVE, A.G. 2000 - Heterogeneidade florística das matas ciliares. In. Rodrigues, R.R.; Leitão Filho, H.F. (ed). Matas ciliares; conservação e recuperação. São Paulo: EDUSP, FAPESP, P.45-75.

SASSI, R.; OLIVEIRA, B. R.; ARAÚJO,M.E.; MOURA G. F.; MELO, J.A. e MELO,G.N. 1997 - Estudo integrado das lagunas costeiras do Estado da Paraíba. Relatório Técnico Final. João Pessoa.

SEMAM (Secretaria Municipal de Meio Ambiente de João Pessoa). 2012 - Plano municipal de recuperação e conservação da mata atlântica de João Pessoa. Prefeitura Municipal de João Pessoa. 100 p. ilus

SILVA JÚMIOR, M. C.; NOGUEIRA, P.E. e FELFILI, J.M. 1998 - Flora Lenhosa das Matas de Galeria no Brasil Central. Boletim do Herbário Ezechias Paulo Heringer 5: 57-76. 